\title{
The far-ultraviolet spectra of two hot PG 1159 stars
}

\author{
K. Werner ${ }^{1}$, T. Rauch ${ }^{1}$, and J. W. Kruk ${ }^{2}$ \\ ${ }^{1}$ Institute for Astronomy and Astrophysics, Kepler Center for Astro and Particle Physics, Eberhard Karls University, Sand 1, \\ 72076 Tübingen, Germany \\ e-mail: werner@astro.uni-tuebingen.de \\ 2 NASA Goddard Space Flight Center, Greenbelt, MD 20771, USA
}

Received 10 May 2016 / Accepted 7 July 2016

\begin{abstract}
PG 1159 stars are hot, hydrogen-deficient (pre-) white dwarfs with atmospheres mainly composed of helium, carbon, and oxygen. The unusual surface chemistry is the result of a late helium-shell flash. Observed element abundances enable us to test stellar evolution models quantitatively with respect to their nucleosynthesis products formed near the helium-burning shell of the progenitor asymptotic giant branch stars. Because of the high effective temperatures $\left(T_{\text {eff }}\right)$, abundance determinations require ultraviolet spectroscopy and non-local thermodynamic equilibrium model atmosphere analyses. Up to now, we have presented results for the prototype of this spectral class and two cooler members ( $T_{\text {eff }}$ in the range $85000-140000 \mathrm{~K}$ ). Here we report on the results for two even hotter stars (PG 1520+525 and PG 1144+005, both with $T_{\text {eff }}=150000 \mathrm{~K}$ ) which are the only two objects in this temperature-gravity region for which useful far-ultraviolet spectra are available, and revisit the prototype star. Previous results on the abundances of some species are confirmed, while results on others ( $\mathrm{Si}, \mathrm{P}, \mathrm{S})$ are revised. In particular, a solar abundance of sulphur is measured in contrast to earlier claims of a strong S deficiency that contradicted stellar evolution models. For the first time, we assess the abundances of $\mathrm{Na}$, Al, and $\mathrm{Cl}$ with newly constructed non-LTE model atoms. Besides the main constituents (He, C, O), we determine the abundances (or upper limits) of N, F, Ne, Na, Al, Si, P, S, Cl, Ar, and Fe. Generally, good agreement with stellar models is found.
\end{abstract}

Key words. stars: abundances - stars: atmospheres - stars: evolution - stars: AGB and post-AGB - white dwarfs

\section{Introduction}

PG 1159 stars are hydrogen-deficient and occupy the hot end of the white dwarf cooling sequence (effective temperatures ranging between $T_{\text {eff }}=75000 \mathrm{~K}$ and $250000 \mathrm{~K}$, Werner \& Herwig 2006; Werner et al. 2014; Werner \& Rauch 2015). Their surfaces are dominated by helium-rich intershell matter dredged up by a late helium-shell flash (thermal pulse, TP). The main atmospheric constituents are helium and carbon $(\mathrm{He}=0.30-0.92, \mathrm{C}=$ 0.08-0.60, mass fractions), often accompanied by large amounts of oxygen (up to 0.20 ). The high abundances of $\mathrm{C}$ and $\mathrm{O}$ are witness to efficient overshoot of intershell convection in asymptotic giant branch (AGB) stars, drawing these elements from the $\mathrm{C}-\mathrm{O}$ stellar core (Herwig et al. 1999; Herwig 2000; Miller Bertolami 2016). Measured overabundances of neon confirm this picture. Our investigation of these stars now concentrates on the trace elements up to the iron group. Quantitative spectroscopic abundance determinations can be compared to predictions from nucleosynthesis calculations in stellar evolution models for AGB stars.

The first comprehensive analysis of trace metal abundances was performed for the prototype of the PG 1159 spectroscopic class, PG 1159-035 ( $T_{\text {eff }}=140000 \mathrm{~K}$, Jahn et al. 2007). Then, two cooler objects from this group were analysed (PG 1424+535 and PG $1707+427, T_{\text {eff }}=110000$ and $85000 \mathrm{~K}$, Werner et al. 2015).

Prerequisites for these types of work are ultraviolet (UV) spectroscopic observations and model atmospheres accounting for departures from local thermodynamic equilibrium (LTE), because of the high effective temperatures. The far-UV spectral range covered by the Far Ultraviolet Spectroscopic Explorer (FUSE, 912-1188 $\AA$ ) is a most rewarding source.
In the present paper, we assess the element abundances of two representatives of this group that are hotter than the prototype, namely $150000 \mathrm{~K}$ : PG $1520+525$ and PG $1144+005$. They are the hottest PG 1159 stars for which useful FUSE data are available. For their analysis we developed new non-LTE model atoms, concentrating on high ionisation stages, particularly for elements not considered previously $(\mathrm{Na}, \mathrm{Mg}, \mathrm{Al}, \mathrm{Cl})$. We strive to identify spectral lines that have not been observed before in any stellar spectrum. The effective temperature of both stars is only rivaled by a few low-gravity PG 1159-type central stars with $T_{\text {eff }}$ up to $170000 \mathrm{~K}$ (K1-16, RX J2117.1+3412, NGC 246, Longmore 4; Werner et al. 2010) for which good FUSE spectra are available. But for a comprehensive quantitative analysis, they require expanding-atmosphere models and the design of model atoms for even higher ionisation stages. In addition to the two program stars, we revisit the prototype itself. It is necessary and useful to analyse a larger number of these stars because, according to stellar models, the abundances of individual trace elements strongly depend on the stellar mass.

\section{The program stars}

Both stars analysed in this paper were discovered in the PalomarGreen Survey (Green et al. 1986). PG 1520+525 was identified as a very hot He-rich degenerate, classified a PG 1159 star, and a parameter estimate based on LTE models was attempted $\left(T_{\text {eff }} \approx 100000 \mathrm{~K}, \log \left(\mathrm{g} / \mathrm{cm} \mathrm{s}^{-2}\right)>6\right.$, Wesemael et al. 1985). PG 1144+005 was classified a PG 1159 star by Basile \& Holberg (1985) and Green et al. (1986). Both objects are non-pulsators (Grauer et al. 1987), in contrast to many other PG 1159 stars, including the prototype ( $\mathrm{GW}$ Vir variables). PG $1520+525$ is 
the central star of an old planetary nebula (PK 085+52 1, Jacoby \& van de Steene 1995).

\section{1. $P G 1520+525$}

The first non-LTE analysis was performed by Werner et al. (1991) using optical spectra. They found $T_{\mathrm{eff}}=140000 \pm$ $14000 \mathrm{~K}, \log g=7.0 \pm 0.5, \mathrm{He}=0.33, \mathrm{C}=0.50, \mathrm{O}=0.17$ (element abundances in mass fractions). Nitrogen lines were not discovered and $\mathrm{N}<4 \times 10^{-3}$ was derived. Utilizing UV spectra taken with the Goddard High Resolution Spectrograph aboard the Hubble Space Telescope, these parameters were improved by Dreizler \& Heber (1998): $T_{\text {eff }}=150000 \pm 7500 \mathrm{~K}, \log g=$ $7.5 \pm 0.5, \mathrm{He}=0.44, \mathrm{C}=0.39, \mathrm{O}=0.17, \mathrm{~N}<1.5 \times 10^{-4}$. The slightly higher temperature was confirmed by the analysis of a Chandra/LETG soft-X-ray spectrum $(150000 \pm 10000 \mathrm{~K}$, Adamczak et al. 2012).

Abundances of further elements were measured from FUSE spectra. $\mathrm{Ne}=0.02$ and $\mathrm{F}=10^{-4}$ was determined (Werner et al. 2004, 2005). Preliminary results for other light element abundances were presented by Reiff et al. (2007): $\mathrm{Si}<3.6 \times 10^{-5}$, $\mathrm{P}=6.4 \times 10^{-6}, \mathrm{~S}=5.0 \times 10^{-5}$. An upper abundance limit for argon of roughly solar was derived $\left(\mathrm{Ar}<8 \times 10^{-5}\right.$, Werner et al. 2007a). From the lack of Fe VII lines, an upper abundance limit of $\mathrm{Fe}<0.3$ solar was estimated (Miksa et al. 2002). Finally, from a fit to newly identified Fe VIII lines, a solar iron abundance was claimed (Werner et al. 2011).

\section{2. $P G 1144+005$}

The first non-LTE analysis was performed by Werner \& Heber (1991) using optical spectra. They found $T_{\text {eff }}=150000 \pm$ $15000 \mathrm{~K}, \log g=6.5 \pm 0.5, \mathrm{He}=0.39, \mathrm{C}=0.58, \mathrm{O}=0.016$. A relatively high nitrogen abundance was determined $(\mathrm{N}=0.015)$.

As in the case of PG $1520+525$, abundances of further elements were measured from FUSE spectra. $\mathrm{Ne}=0.02$ was found from an analysis, including optical spectra (Werner et al. 2004) and $\mathrm{F}=10^{-5}$ was determined (Werner et al. 2005). Preliminary results for other light element abundances were presented by Reiff et al. (2007): $\mathrm{Si}<7.3 \times 10^{-5}, \mathrm{P}=6.4 \times 10^{-6}, \mathrm{~S}=5.0 \times 10^{-5}$. An upper abundance limit for argon of roughly solar was derived ( $\mathrm{Ar}<8 \times 10^{-5}$, Werner et al. 2007a). From a fit to Fe VIII lines in FUSE spectra, a solar iron abundance was determined (Werner et al. 2011).

\section{Observations and line identifications}

The raw FUSE data for the program stars were retrieved from the Mikulski Archive for Space Telescopes (MAST). The observation IDs were P1320201 for PG 1144+005, and P1320101, F0200101, F0200102 for PG 1520+525. Both were observed through the LWRS spectrograph aperture, and the data were obtained in time-tag mode. We describe the data reduction in some detail because the previous reductions in our earlier work did not go past the point of coaligning spectra from exposures for the eight individual channels separately, whereas now the spectra from the individual channels were combined into a single spectrum.

The exposures obtained in the observations P1320101 and P1320201 were photometric in all channels, with exposure-toexposure flux variations under $1 \%$, indicating good alignment, and consistent to within a few percent when comparing one channel to another. Only one exposure was successful in each of F0200101 and F0200102, and pointing was unstable for portions of these exposures; the resulting spectra were normalized to match the earlier P1320101 data.

Raw data were processed twice with CalFUSE v3.2.3: once with screening parameters set to extract data only during orbital night, and once to extract data during orbital day. Zero-point offsets in the wavelength scale were adjusted for each exposure by shifting each spectrum to coalign narrow interstellar absorption features. The individual exposures from each observation were then combined to form composite day and night spectra for each channel. The day and night spectra were then compared at the locations of all the known airglow emission lines. If the day spectra showed any excess flux in comparison to the night spectra at those wavelengths, the corresponding pixels in the day spectra were flagged as bad and were not included in subsequent processing. Significant airglow was only present during orbital night at Lyman $\beta$, and faint emission can be discerned at Lyman $\gamma$. This residual emission would affect fits to the interstellar absorption at these wavelengths, but has no impact on any of the analyses we present.

Some additional customized processing was performed for PG $1520+525$. The first $\approx 350 \mathrm{~s}$ of the first and third exposures were lost to the limb-angle screening with the default processing settings. However, an examination of the data showed that the violation of the limb angle constraint was slight, so we modified the constraint check to regain over $700 \mathrm{~s}$ of exposure time. Similarly, $315 \mathrm{~s}$ were lost to burst screening in detector $2 \mathrm{~A}$ during F0200101, and losses in F0200102 ranged from $135 \mathrm{~s}$ in detector $2 \mathrm{~B}$ to $432 \mathrm{~s}$ in detector $1 \mathrm{~A}$. An examination of the raw data showed no obvious background enhancement from the event burst, so the processing was performed with a higher burstdetection threshold to regain the lost exposure time. The net exposure time was $6830 \mathrm{~s}$ for PG 1144+005, with just slight variations from channel to channel, and ranged from a low of $7758 \mathrm{~s}$ in the $\mathrm{SiC} 1 \mathrm{~b}$ spectrum to $8600 \mathrm{~s}$ in the LiF1a spectrum for PG $1520+525$.

The final step was to combine the spectra from the four instrument channels into a single composite spectrum. Because of residual distortions in the wavelength scale in each channel, additional shifts of localized regions of each spectrum were required to coalign the spectra; these shifts were typically only 12 pixels. Bad pixels resulting from detector defects were flagged at this point and excluded from further processing. Finally, the spectra were resampled onto a common wavelength scale and combined, weighting by signal-to-noise ratio $(\mathrm{S} / \mathrm{N})$ on a pixelby-pixel basis.

The final spectrum has $S / N=13-23$ per $0.013 \AA$ pixel for PG $1520+525$, and 14-26 per pixel for PG $1144+005$. The effects of fixed-pattern noise are minimized by the fact that the positions of the spectra on the detectors varied during each observation, and by the fact that nearly every wavelength bin was sampled by at least two different detectors.

The FUSE spectra of both program stars (Figs. 1 and 2) are quite similar to that of the prototype PG 1159-035, as can be expected from the similar atmospheric parameters. They are dominated by broad lines from He II, C IV, and O VI, and exhibit some lines of light metals that will be discussed below (see Jahn et al. 2007, for detailed line lists established for the prototype). In PG $1520+525$, we also identify lines of O v, which are seen in PG 1159-035, too, but which were not identified in previous work (Jahn et al. 2007). Detailed information about O V lines was compiled for two cool PG 1159 stars (Werner et al. 2015). O V is not detected in PG 1144+005. Ionisation is shifted to higher stages because the surface gravity is one dex lower than 


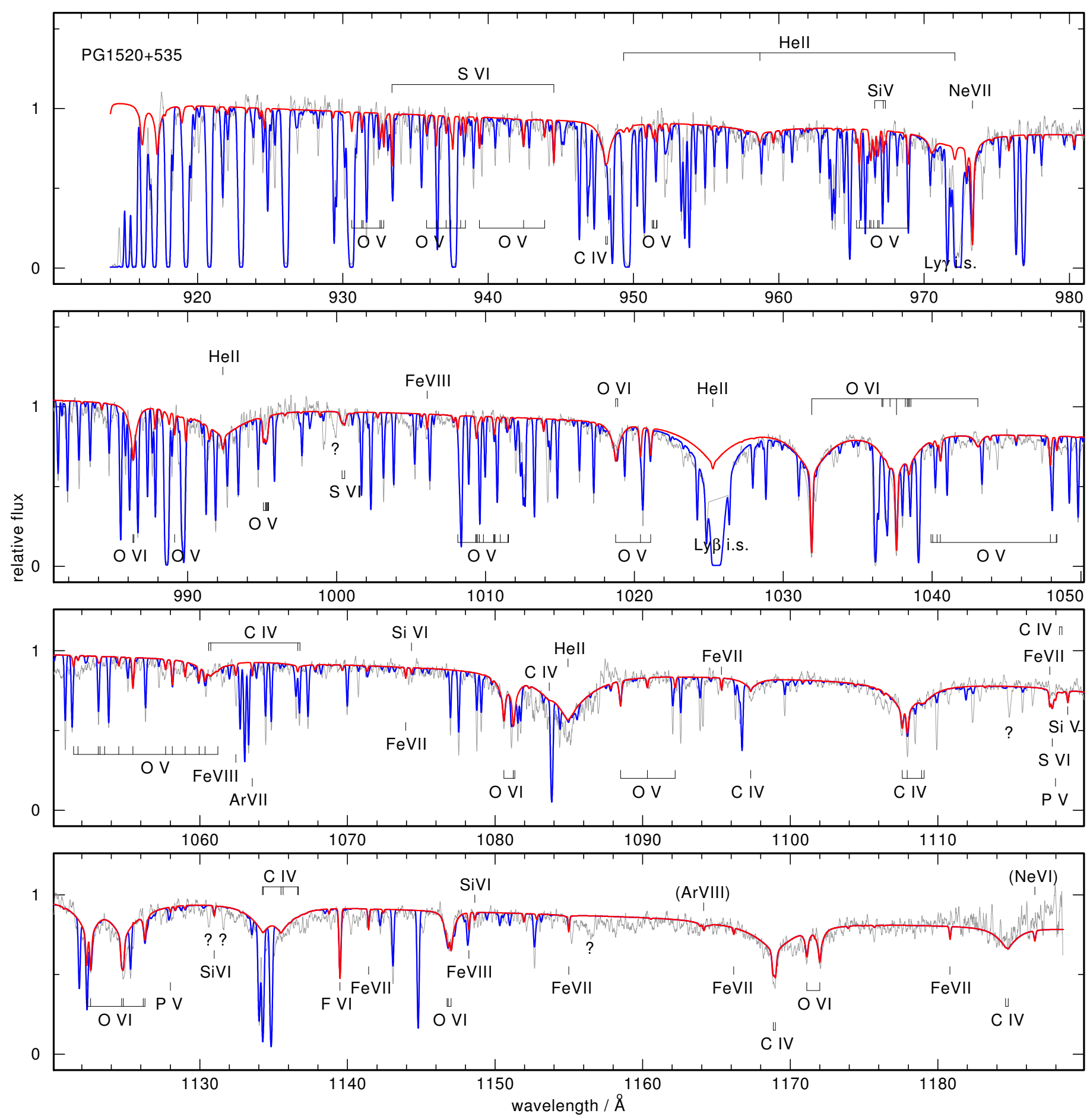

Fig. 1. FUSE spectrum of PG $1520+525$ (thin black line) compared to a photospheric model spectrum (thick red line) with the finally adopted parameters as listed in Table 1. Prominent photospheric spectral lines are identified. Uncertain identifications are given in brackets. Unidentified, probably photospheric, lines are indicated by question marks. Geocoronal emission in the center of Lyman $\beta$ was cut out. Overplotted in blue is the same model including ISM lines.

that of PG $1520+525$. We also see iron lines in PG 1159-035 and our program stars (Fe VII and Fe VIII, Werner et al. 2011). Other species are discussed in detail below. The FUSE spectra shown in this paper were wavelength shifted such that the photospheric lines are at rest wavelengths according to the measured radial velocities of $15 \pm 5 \mathrm{~km} \mathrm{~s}^{-1}$ and $10 \pm 5 \mathrm{~km} \mathrm{~s}^{-1}$ for PG 1144+005 and PG $1520+525$.

To identify lines from the interstellar medium (ISM) and to judge their potential contamination of photospheric lines, we used the program OWENS (Hébrard et al. 2002; Hébrard \& Moos 2003). This can consider different clouds with individual radial and turbulent velocities, temperatures, column densities, and chemical compositions. In the FUSE observations of both stars, we identified ISM lines of H I, D I, $\mathrm{H}_{2}$, C I-III, N III, O I, Si II, P II, S III, Ar I, and Fe II. The blue graphs in Figs. 1 and 2 show the photospheric models including the ISM lines.

Interstellar reddening is $E(B-V)=0.015 \pm 0.010$ for both stars, determined by comparing model fluxes normalized to 


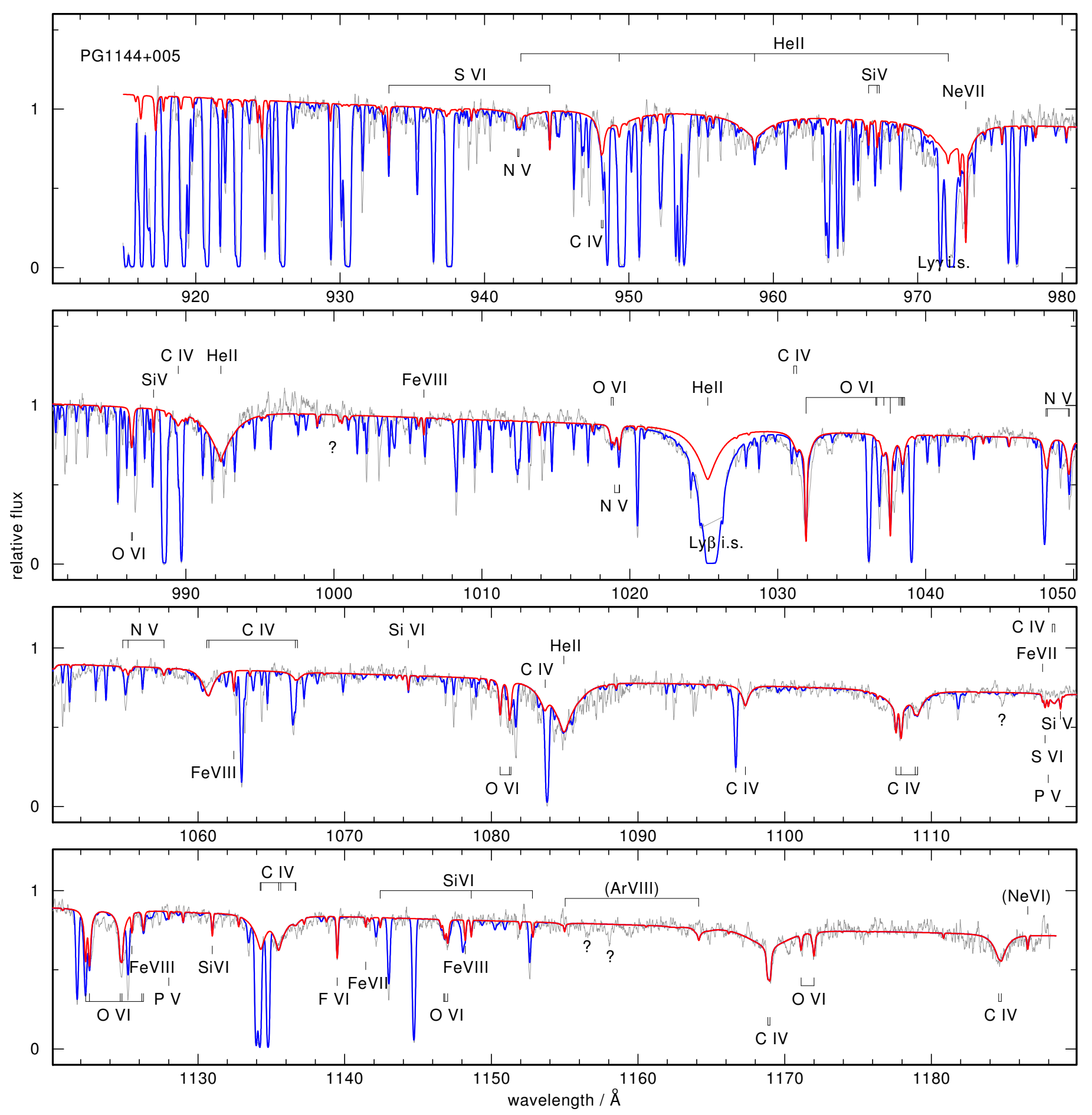

Fig. 2. As Fig. 1, for PG 1144+005.

measured 2MASS infrared colours $\left(K_{\mathrm{s}}=16.282\right.$ for PG 1144+005 and $J=16.381$ for PG $1520+525$, Debes et al. 2011) with the continuum flux and spectral shape of the FUSE spectra.

\section{Model atoms and model atmospheres}

We used the Tübingen Model Atmosphere Package (TMAP) to compute non-LTE, plane-parallel, line-blanketed atmosphere models in radiative and hydrostatic equilibrium (Werner \& Dreizler 1999; Werner et al. 2003). They include the three most abundant elements, namely He, C, and O. All other species were treated, one by one, as trace elements, i.e., keeping the atmospheric structure fixed. In the same manner, an extended model atom for $\mathrm{C}$ was introduced, meaning that non-LTE population numbers were computed for highly-excited levels, which were treated in LTE during the preceding modelatmosphere computations. Table 2 summarizes the number of considered non-LTE levels and radiative transitions between them. All model atoms were built from the publicly available Tübingen Model Atom Database (TMAD ${ }^{1}$, constructed as

1 http://astro.uni-tuebingen.de/ TMAD 
K. Werner et al.: The far-ultraviolet spectra of two hot PG 1159 stars

Table 1. Final adopted parameters for the program stars PG 1144+005 and PG $1520+525$.

\begin{tabular}{rrrrr}
\hline \hline & PG 1144+005 & PG 1520+525 & PG 1159-035 & Sun $^{a}$ \\
\hline$T_{\text {eff }} / \mathrm{K}$ & 150000 & 150000 & 140000 & \\
$\log g$ & 6.5 & 7.5 & 7.0 & \\
$\mathrm{H}$ & $<0.10$ & $<0.10$ & $<0.02$ & 0.74 \\
$\mathrm{He}$ & 0.38 & 0.43 & 0.33 & 0.25 \\
$\mathrm{C}$ & 0.57 & 0.38 & 0.48 & $2.4 \times 10^{-3}$ \\
$\mathrm{~N}$ & 0.015 & $<1.5 \times 10^{-4}$ & 0.001 & $6.9 \times 10^{-4}$ \\
$\mathrm{O}$ & 0.016 & 0.17 & 0.17 & $5.7 \times 10^{-3}$ \\
$\mathrm{~F}$ & $1.0 \times 10^{-5}$ & $1.0 \times 10^{-4}$ & $3.2 \times 10^{-6}$ & $5.0 \times 10^{-7}$ \\
$\mathrm{Ne}$ & 0.02 & 0.02 & 0.02 & 0.0013 \\
$\mathrm{Na}$ & $<0.01$ & - & - & $2.9 \times 10^{-5}$ \\
$\mathrm{Al}$ & $<1.0 \times 10^{-3}$ & $<3.2 \times 10^{-4}$ & $<3.2 \times 10^{-4}$ & $5.6 \times 10^{-5}$ \\
$\mathrm{Si}$ & $6.6 \times 10^{-4}$ & $6.6 \times 10^{-4}$ & $3.6 \times 10^{-4}$ & $6.6 \times 10^{-4}$ \\
$\mathrm{P}$ & $<3.0 \times 10^{-5}$ & $<3.0 \times 10^{-5}$ & $<6.4 \times 10^{-6}$ & $5.8 \times 10^{-6}$ \\
$\mathrm{~S}$ & $1.0 \times 10^{-4}$ & $3.1 \times 10^{-4}$ & $3.1 \times 10^{-4}$ & $3.1 \times 10^{-4}$ \\
$\mathrm{Cl}$ & $<1.0 \times 10^{-3}$ & $<1.0 \times 10^{-3}$ & $<1.0 \times 10^{-3}$ & $8.2 \times 10^{-6}$ \\
$\mathrm{Ar}$ & $<8.0 \times 10^{-5}$ & $<8.0 \times 10^{-5}$ & $<3.2 \times 10^{-5}$ & $7.3 \times 10^{-5}$ \\
$\mathrm{Fe}$ & $1.3 \times 10^{-3}$ & $1.3 \times 10^{-3}$ & $1.3 \times 10^{-3}$ & $1.3 \times 10^{-3}$ \\
\hline
\end{tabular}

Notes. The results are compared to the prototype of the PG 1159 spectral class (PG 1159-035) and the Sun. Abundances in mass fractions and surface gravity $g$ in $\mathrm{cm} \mathrm{s}^{-2}$. ${ }^{(a)}$ Solar abundances from Asplund et al. (2009). Parameters for PG 1159-035 from Jahn et al. (2007) and references therein, except for the abundances of Fe (from Werner et al. 2011) and $\mathrm{Al}, \mathrm{S}, \mathrm{Cl}$ (this work). Upper limits for $\mathrm{H}$ in PG 1144+005, PG 1520+525, and PG 1159-035 from Werner \& Heber (1991), Werner et al. (1991, 1996).

part of the German Astrophysical Virtual Observatory, GAVO), which is comprised of data from different sources, namely Bashkin \& Stoner (1975), the databases of the National Institute of Standards and Technology (NIST ${ }^{2}$ ), the Opacity Project ${ }^{3}$ (OP, Seaton et al. 1994), CHIANTI ${ }^{4}$ (Dere et al. 1997; Landi et al. 2013), as well as the Kentucky Atomic Line List ${ }^{5}$. We put significant effort into the development and testing of model atoms for species that had not been investigated before in hot PG1159 stars $(\mathrm{Na}, \mathrm{Mg}, \mathrm{Al}, \mathrm{Cl})$ with an emphasis on the highest ionization stages.

For iron we used a statistical approach, employing typically seven superlevels per ion linked by superlines, together with an opacity sampling method (Anderson 1989; Rauch \& Deetjen 2003). Ionization stages Fe VI-IX augmented by a single, ground-level stage Fe X were considered. We used the complete line list of Kurucz (so-called LIN lists, comprising about $1.2 \times 10^{6}$ lines of the considered ions; Kurucz 1991, 2009, 2011) for the computation of the non-LTE population numbers, and the so-called POS lists (that include only the subset of lines with well known, experimentally observed line positions) for the final spectrum synthesis.

\section{Results}

For the abundance analysis, we adopted the effective temperature and surface gravity values of both stars that had been determined in previous work. We found no hint from the FUSE spectra that these values need improvement. This is corroborated by the relative strengths of $\mathrm{Si} \mathrm{V}$ and $\mathrm{Si}$ VI lines which are well matched by the models. The new detection of many $\mathrm{OV}$ lines

\footnotetext{
2 http://www.nist.gov/pml/data/asd.cfm

http://cdsweb.u-strasbg.fr/topbase/topbase.html

http://www . chiantidatabase.org

http://www.pa.uky.edu/ peter/atomic
}

Table 2. Number of non-LTE levels and lines of model ions used for line formation calculations of metals.

\begin{tabular}{ccccccc}
\hline \hline & III & IV & V & VI & VII & VIII \\
\hline $\mathrm{C}$ & 6,4 & 54,279 & & & & \\
$\mathrm{~N}$ & & 6,4 & 54,297 & & & \\
$\mathrm{O}$ & & 83,637 & 105,671 & 54,280 & & \\
$\mathrm{~F}$ & & 1,0 & 16,32 & 18,36 & 14,28 & \\
$\mathrm{Ne}$ & & & & 92,687 & 103,761 & 77,510 \\
$\mathrm{Na}$ & & 49,39 & 52,229 & 65,301 & 29,80 & \\
$\mathrm{Mg}$ & & 31,93 & 52,175 & 26,55 & 46,147 & \\
$\mathrm{Al}$ & 1,0 & 15,20 & 30,87 & 43,126 & 43,160 & \\
$\mathrm{Si}$ & & 30,102 & 25,59 & 45,193 & 61,138 & \\
$\mathrm{P}$ & & 21,9 & 18,12 & & & \\
$\mathrm{~S}$ & & & 39,107 & 25,48 & 38,120 & \\
$\mathrm{Cl}$ & 34,107 & 20,34 & 13,10 & 36,94 & 25,64 & \\
$\mathrm{Ar}$ & & & & 8,8 & 40,130 & 41,199
\end{tabular}

Notes. First and second number of each table entry denote the number of levels and lines, respectively. Not listed for each element is the highest considered ionisation stage, which comprises its ground state only. See text for the treatment of iron.

in PG $1520+525$ together with O VI lines additionally confirms that the basic photospheric parameters, $T_{\text {eff }}$ and $\log g$, are well established. The same holds for the abundance of the main atmospheric constituents ( $\mathrm{He}, \mathrm{C}$, and $\mathrm{O}$ ).

In the following, we discuss the abundance determinations for trace elements in detail. Results for the two program stars are given in Table 1 and compared to the prototype PG 1159-035. They are also displayed graphically in Fig. 3 in comparison to PG 1159-035 and two other previously analysed PG 1159 stars (PG 1707+427 and PG 1424+535, Werner et al. 2015). Typical errors are 0.3 dex.

Nitrogen. The relatively high $\mathrm{N}$ abundance in PG 1144+005 was found from optical spectroscopy and cannot be further assessed with the FUSE spectrum. The strongest lines in the model are N v 4d-6f and 4f-6g at 1048.2 $\AA$ and $1049.7 \AA$, but they are blended by strong interstellar lines in the observation. The next strongest feature is the $4 \mathrm{p}-6 \mathrm{~d}$ doublet at $1019.0 / 1019.3 \AA$, but it is blended by a strong photospheric O VI line and an interstellar line. For the same reason, the upper $\mathrm{N}$ limit for PG $1520+525$, derived previously from the $\mathrm{N} V$ resonance doublet, cannot be improved.

Fluorine, neon, argon, iron. The previously determined abundances of F, Ne, Ar (upper limits), and Fe in both stars are confirmed.

A weak Ne VI line appearing in the models at $1186.6 \AA$ is possibly present in PG $1144+005$. The line is not listed in the NIST and Kentucky databases but in the CHIANTI database. The oscillator strength given there is $f=0.068$, while we use the larger OP value of $f=0.097$ in our models. A stronger $\mathrm{Ne}$ VI line in the models, that is also listed in the NIST database, is seen at $972.93 \AA$ (near the Ne VII $973.33 \AA$ line). However, it is blended by strong interstellar lines.

The model for PG 1144+005 displays the 5f-6g and 5g-6h lines of Ar VIII at 1155.0 and 1164.1 $\AA$ (Hegazy et al. 1997). However, their wavelength positions are not well enough known $( \pm 0.84$ and $\pm 1.2 \AA$, Kentucky database) to identify them with features in the observed spectra. We speculated about the presence of these argon lines in the hottest PG 1159 stars (e.g., K116, Werner et al. 2007b). 


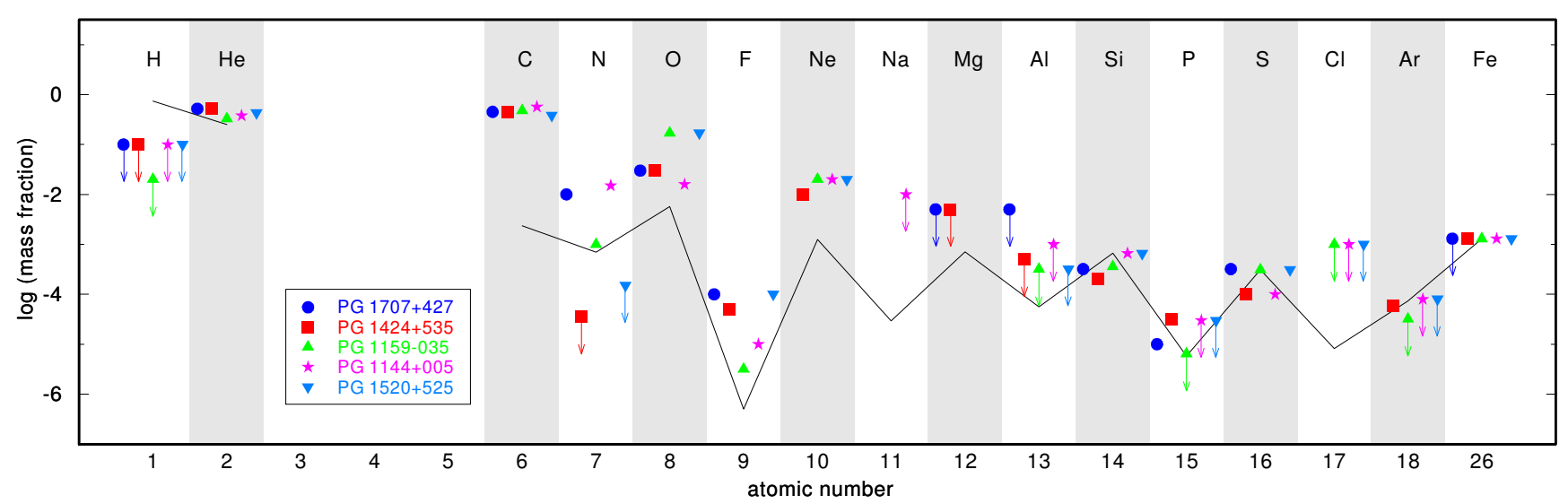

Fig. 3. Element abundances measured in five PG 1159 stars. Upper limits are indicated by arrows. Data for our two program stars and PG $1159-035$ are from Table 1. Data for PG 1707+427 and PG 1424+535 are from Werner et al. (2015) except for the H abundances (from Werner et al. 1991). The black solid line indicates solar abundances.

Sodium. Our models predict detectable, i.e., strong enough lines of $\mathrm{NaV}$ and $\mathrm{Na}$ VI only at high abundances of the order 0.01 . Even worse, the line positions are not sufficiently accurate. In the model for PG 1144+005, a weak Na VII line with well known wavelength position (1114.08 $\mathrm{A}$, NIST) appears, but no tight abundance limit can be derived $(\mathrm{Na}<0.01)$. Neither the model for PG $1520+525$ exhibits this line because of its higher gravity, nor the model for PG 1159-035, because of its lower temperature.

Magnesium. Our models predict Mg V lines which should be detectable if the abundance is higher than about $10^{-3}$. Unfortunately, these lines are not listed in NIST and Kentucky databases so that their wavelength position seems uncertain. Consequently, not even an upper limit can be derived. The same holds for a single Mg VII line in the model for PG 1144+005.

Aluminum. Our models predict weak lines from $\mathrm{Al} \mathrm{V}$ and $\mathrm{Al}$ VI that can be used to assess the $\mathrm{Al}$ abundance. The strongest computed lines of these ions are Al V 1068.26 $\AA$ and 1088.67 $\AA$, that are the strongest components of ${ }^{4} \mathrm{P}^{\mathrm{o}}-{ }^{4} \mathrm{D}$ and ${ }^{4} \mathrm{D}^{\mathrm{o}}-{ }^{4} \mathrm{~F}$ multiplets, and Al VI $1056.00 \AA$, the strongest component of a ${ }^{5} \mathrm{P}-{ }^{5} \mathrm{D}^{\mathrm{o}}$ multiplet. In PG $1520+525$, weak line features are at these wavelength positions. However, we regard these identifications as uncertain. An upper limit of $\mathrm{Al}<3.2 \times 10^{-4}$ (about six times solar) can be derived. In the model for PG 1144+005, the ionisation of $\mathrm{Al}$ shifts to higher stages because of the lower gravity (hence lower particle densities). Therefore, the derived upper limit is less strict, namely $\mathrm{Al}<1.0 \times 10^{-3}$. The $\mathrm{Al}$ abundance in the prototype, PG 1159-035, has hitherto not been investigated. Using an appropriate model atmosphere, we derive $\mathrm{Al}<3.2 \times 10^{-4}$.

Silicon. We identified a few weak lines from Siv (975.8, $1118.8 \AA$ A) and Si VI (1131.0, 1142.4, 1148.6, 1152.8 ̊). We obtain best-model fits at a solar abundance value $\left(\mathrm{Si}=6.6 \times 10^{-4}\right)$ for both stars, hence, we do not confirm the stricter upper limits derived by Reiff et al. (2007) from the absence of Si IV lines in the FUSE observations.

Phosphorus. From the nondetection of the $\mathrm{PV}$ resonance doublet $\left(1118,1128 \AA\right.$ ), we derive an upper limit of $\mathrm{P}<3.0 \times 10^{-5}$

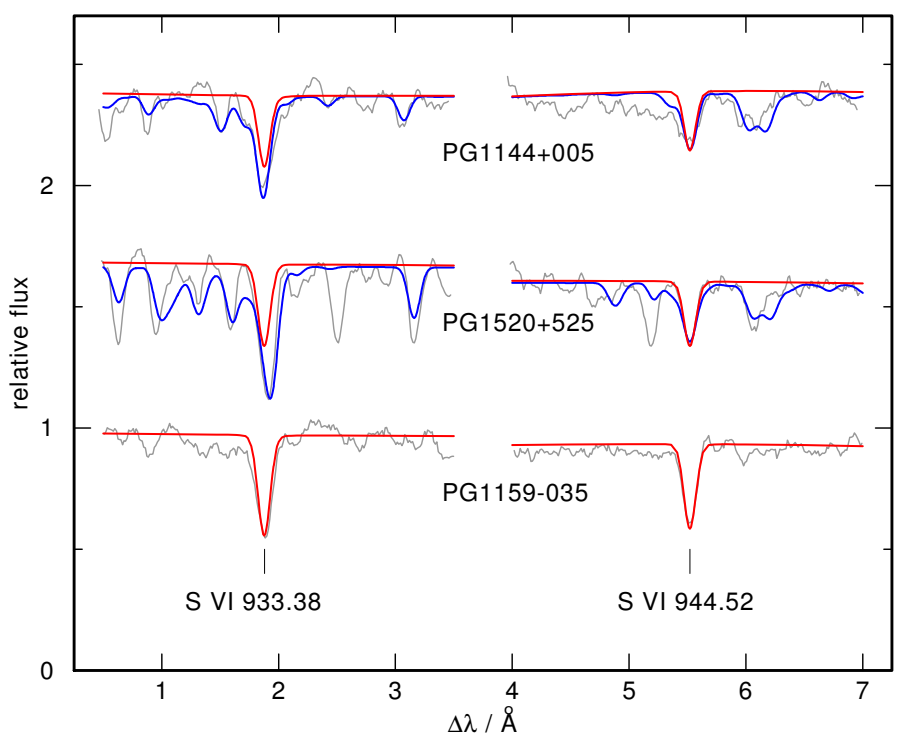

Fig. 4. Model fits to the S VI resonance doublet in the two program stars and in the prototype PG 1159-035, with parameters as given in Table 1. In PG 1144+005 and PG 1520+525, the $933.38 \AA$ component is blended by an interstellar $\mathrm{H}_{2}$ line. Red curves: pure photospheric spectrum. Blue curves: ISM lines included in the model (not for PG 1159-035).

for both stars. This value is more conservative than the limits claimed by Reiff et al. (2007).

Sulphur. From the S VI 933/944 $\AA$ resonance doublet (Fig. 4) and from two much weaker subordinate lines at $1000.5 \AA$ and $1117.8 \AA$, we find $S=1.0 \times 10^{-4}$ and $3.1 \times 10^{-4}$ for PG $1144+005$ and PG $1520+525$, respectively, being solar within error limits. This result is in stark contrast to the earlier results reported by Reiff et al. (2007) that indicate a one dex subsolar abundance for these stars. We discovered that the low $\mathrm{S}$ values resulted from an error in the S VI model atom (wrong statistical weights for two energy levels) employed for the computation of the non-LTE population numbers.

We mention that the error in the model atom also affected the sulphur abundance determination in the prototype PG 1159-035 in our earlier work (Jahn et al. 2007). A surprisingly low value of just $2 \%$ solar was found, whereas a reanalysis with our corrected model atom arrives at a solar abundance. 
Table 3. Asteroseismic, spectroscopic, and initial masses of the five considered PG 1159 stars.

\begin{tabular}{llll}
\hline \hline Star & $M_{\text {puls }} / M_{\odot}$ & $M_{\text {spec }} / M_{\odot}$ & $M_{\text {init }} / M_{\odot}{ }^{(e)}$ \\
\hline PG 1424+535 & - & $0.51_{-0.01}^{+0.07(c)}$ & $\approx 1-2$ \\
PG 1707+427 & $0.542_{-0.012}^{+0.014(a)}$ & $0.53_{-0.03}^{+0.17(c)}$ & $\approx 1-3$ \\
PG 1159-035 & $0.565_{-0.009}^{+0.025(b)}$ & $0.54_{-0.01}^{+0.05(c)}$ & $\approx 1-2$ \\
PG 1144+005 & - & $0.56_{-0.03}^{+0.07(d)}$ & $\approx 1-2.5$ \\
PG 1520+525 & - & $0.62_{-0.08}^{+0.15(d)}$ & $\approx 1-4$ \\
\hline
\end{tabular}

References. (a) Córsico et al. (2008); (b) Córsico et al. (2009); ${ }^{(c)}$ Werner \& Rauch (2014); ${ }^{(d)}$ this work; ${ }^{(e)}$ estimated from initial-tofinal mass relation of Weidemann (2000) using $M_{\text {spec }}$.

Chlorine. Lines of $\mathrm{Cl}$ VII enable us to put an upper abundance limit, though it is not very tight. $\mathrm{Cl}$ VII is a one-valence-electron system. The strongest predicted lines are from the $4 d-5 p$ transition (997.03 $\AA$ and $999.32 \AA$ ) and the $5 \mathrm{f}-7 \mathrm{~g}$ transition at $944.1 \AA$ but, for the latter, the wavelength uncertainty is too large $(0.57 \AA$; Kentucky database). From the former we find $\mathrm{Cl}<10^{-3}$ for both program stars as well as for PG 1159-035.

\section{Summary and discussion}

We have analysed FUV spectra of two hot PG 1159 stars (PG 1144+005 and PG 1520+525, both $T_{\text {eff }}=150000 \mathrm{~K}$ ) with the focus on element abundance determinations. Previously obtained values for $T_{\text {eff }}$ and $\log g$ were confirmed. With our improved model atmospheres, we additionally derived new findings on the prototype itself (PG 1159-035; $140000 \mathrm{~K}$ ). The results for these three objects are summarized in Table 1. In Fig. 3, the element abundances are displayed, together with the results for two cooler PG 1159 stars (PG 1707+427 and PG 1424+535 with 85000 and $110000 \mathrm{~K}$; Werner \& Rauch 2014). Taken together, these five objects represent the only PG 1159 stars with comprehensive element abundance determinations.

For the comparison of the results with evolutionary models, stellar masses are relevant. In Table 3 we list the spectroscopic masses derived by comparing them with the observed values of $T_{\text {eff }}$ and $\log g$ with evolutionary tracks by Althaus et al. (2009). For the two pulsators in the sample, asteroseismic masses are also available, being in good agreement with the spectroscopic values. Also listed are the masses of the main-sequence progenitors, as estimated from the initial-to-final mass relation of Weidemann (2000), taking into account the uncertainties of the spectroscopic masses.

The previously determined abundances of the main atmospheric constituents $(\mathrm{He}, \mathrm{C}, \mathrm{O}, \mathrm{Ne}$ ) in our two program stars were confirmed. The same holds for N, F, Ar, and Fe. The investigation on $\mathrm{Si}, \mathrm{P}$, and $\mathrm{S}$ gave improved results compared to earlier, preliminary work. For the first time, we have assessed the abundances of $\mathrm{Na}, \mathrm{Al}$, and $\mathrm{Cl}$, and derived upper limits.

We used our model atmosphere grid to reassess the abundances of trace elements in the prototype PG 1159-035. The main result is an improved $\mathrm{S}$ abundance, which is solar in contrast to the strong $\mathrm{S}$ depletion ( 0.02 solar) claimed by Jahn et al. (2007), which was caused by an error in the model atom. We also derived upper limits for $\mathrm{Na}, \mathrm{Al}$, and $\mathrm{Cl}$ for this star.

The results can be compared to evolutionary models by Shingles \& Karakas (2013), who presented intershell abundances for stars with initial masses of $1.8-6 M_{\odot}$. For PG 1159-035, the authors found good agreement for the species investigated ( $\mathrm{He}, \mathrm{C}, \mathrm{O}, \mathrm{F}, \mathrm{Ne}, \mathrm{Si}, \mathrm{P}, \mathrm{S}, \mathrm{Fe})$ with the exception of sulphur. As pointed out, this discrepancy is now resolved. For the two cool PG 1159 stars mentioned above, good agreement of observed and predicted element abundances was found (Werner \& Rauch 2014). As to our two program stars, all element abundances are similar to those found for the other three PG 1159 stars (Fig. 3) and, hence, confirm the evolutionary models. For a thorough discussion, we can therefore refer to Werner \& Rauch (2014), however, N, Na, Al, and Cl deserve particular attention here. The $\mathrm{Na}$ and $\mathrm{Cl}$ abundances in PG 1159 stars were examined for the first time.

PG 1144+005 is remarkable because of its relatively large N abundance. This phenomenon is shared by some other PG 1159 stars (like the mentioned cooler object PG 1707+427) and is attributed to the fact that these objects suffered their last thermal pulse when they already were white dwarfs (so-called very late thermal pulse, VLTP).

Only for PG 1144+005 could a useful upper limit for the sodium abundance be derived, which is 341 times oversolar. Karakas \& Shingles (priv. comm.) report that the $\mathrm{Na}$ intershell abundance at the last thermal pulse in the 1.8 and $3 M_{\odot}$ models presented in Shingles \& Karakas (2013) has increased considerably to 23.1 and 18.1 times oversolar after the last thermal pulse. This is still well below our detection limit.

For aluminum, upper limits of 18 and 6 times solar were derived for PG $1144+005$ and PG $1520+525$. The Al intershell abundance at the last thermal pulse in the mentioned 1.8 and $3 M_{\odot}$ models is 1.5 and 2 times the initial solar value. Hence, the rather modest production of $\mathrm{Al}$ is confirmed by our result.

For the two program stars, as well as for PG 1159-035, only a high upper limit of $\mathrm{Cl}=10^{-3}$ was derived, which is about two dex oversolar. The intershell abundance in the $1.8 M_{\odot}$ and $3 M_{\odot}$ models has increased to 2.3 and 2.1 times oversolar after the last thermal pulse, i.e., well below our detection limit.

To summarize, the abundances of elements up to iron in PG 1159 stars is in good agreement with intershell abundances predicted by stellar-evolution models. For a number of interesting species $(\mathrm{Na}, \mathrm{Al}, \mathrm{Cl})$, however, only upper limits can be derived by spectral analyses. It turned out that for the relevant ionisation stages (IV-VII), precise atomic data is lacking, impeding line identifications. This obstacle can only be overcome by laboratory measurements. The detailed investigation into the trace element abundances as a function of stellar mass is additionally hindered by the relatively large uncertainty in the spectroscopic mass determination, which itself is due to the uncertainty of the surface gravity measurement $( \pm 0.5 \mathrm{dex})$. Considerable improvement is expected from accurate parallaxes that will be provided by Gaia.

Acknowledgements. We thank Amanda Karakas and Luke Shingles for reporting unpublished results to us. T. Rauch is supported by the German Aerospace Center (DLR) under grant 50 OR 1507. The TMAD service (http://astro-uni-tuebingen.de/ TMAD) used to compile atomic data for this paper was constructed as part of the activities of the German Astrophysical Virtual Observatory. This research has made use of the SIMBAD database, operated at CDS, Strasbourg, France, and of NASA's Astrophysics Data System Bibliographic Services. Some of the data presented in this paper were obtained from the Mikulski Archive for Space Telescopes (MAST). This work had been done using the profile fitting procedure OWENS, developed by M. Lemoine and the FUSE French Team.

\section{References}

Adamczak, J., Werner, K., Rauch, T., et al. 2012, A\&A, 546, A1

Althaus, L. G., Panei, J. A., Miller Bertolami, M. M., et al. 2009, ApJ, 704, 1605 Anderson, L. S. 1989, ApJ, 339, 558 
Asplund, M., Grevesse, N., Sauval, A. J., \& Scott, P. 2009, ARA\&A, 47, 481

Bashkin, S., \& Stoner, J. O. 1975, Atomic energy levels and Grotrian Diagrams - Vol. 1: Hydrogen I - Phosphorus XV; Vol. 2: Sulfur I - Titanium XXII (Amsterdam: North Holland Publishing Co.)

Basile, J., \& Holberg, J. B. 1985, BAAS, 17, 838

Córsico, A. H., Althaus, L. G., Kepler, S. O., Costa, J. E. S., \& Miller Bertolami, M. M. 2008, A\&A, 478, 869

Córsico, A. H., Althaus, L. G., Miller Bertolami, M. M., \& García-Berro, E. 2009, A\&A, 499, 257

Debes, J. H., Hoard, D. W., Wachter, S., Leisawitz, D. T., \& Cohen, M. 2011 ApJS, 197, 38

Dere, K. P., Landi, E., Mason, H. E., Monsignori Fossi, B. C., \& Young, P. R. 1997, A\&AS, 125, 149

Dreizler, S., \& Heber, U. 1998, A\&A, 334, 618

Grauer, A. D., Bond, H. E., Liebert, J., Fleming, T. A., \& Green, R. F. 1987, ApJ 323,271

Green, R. F., Schmidt, M., \& Liebert, J. 1986, ApJS, 61, 305

Hébrard, G., \& Moos, H. W. 2003, ApJ, 599, 297

Hébrard, G., Friedman, S. D., Kruk, J. W., et al. 2002, Planet. Space Sci., 50, 1169

Hegazy, H., Büscher, S., Kunze, H.-J., \& Wrubel, T. 1997, J. Quant. Spectr. Rad Transf., 58, 627

Herwig, F. 2000, A\&A, 360, 952

Herwig, F., Blöcker, T., Langer, N., \& Driebe, T. 1999, A\&A, 349, L5

Jacoby, G. H., \& van de Steene, G. 1995, AJ, 110, 1285

Jahn, D., Rauch, T., Reiff, E., et al. 2007, A\&A, 462, 28

Kurucz, R. L. 1991, in NATO ASIC Proc. 341: Stellar Atmospheres - Beyond Classical Models, eds. L. Crivellari, I. Hubeny, \& D. G. Hummer, 441

Kurucz, R. L. 2009, in AIP Conf. Ser. 1171, eds. I. Hubeny, J. M. Stone, K. MacGregor, \& K. Werner, 43

Kurucz, R. L. 2011, Canad. J. Phys., 89, 417
Landi, E., Young, P. R., Dere, K. P., Del Zanna, G., \& Mason, H. E. 2013, ApJ, 763,86

Miksa, S., Deetjen, J. L., Dreizler, S., et al. 2002, A\&A, 389, 953

Miller Bertolami, M. M. 2016, A\&A, 588, A25

Rauch, T., \& Deetjen, J. L. 2003, in Stellar Atmosphere Modeling, eds. I. Hubeny, D. Mihalas, \& K. Werner, ASP Conf. Ser., 288, 103

Reiff, E., Jahn, D., Rauch, T., et al. 2007, in 15th European Workshop on White Dwarfs, eds. R. Napiwotzki, \& M. R. Burleigh, ASP Conf. Ser., 372, 237

Seaton, M. J., Yan, Y., Mihalas, D., \& Pradhan, A. K. 1994, MNRAS, 266, 805

Shingles, L. J., \& Karakas, A. I. 2013, MNRAS, 431, 2861

Weidemann, V. 2000, A\&A, 363, 647

Werner, K., \& Dreizler, S. 1999, J. Comput. Appl. Math., 109, 65

Werner, K., \& Heber, U. 1991, A\&A, 247, 476

Werner, K., \& Herwig, F. 2006, PASP, 118, 183

Werner, K., \& Rauch, T. 2014, A\&A, 569, A99

Werner, K., \& Rauch, T. 2015, A\&A, 584, A19

Werner, K., Heber, U., \& Hunger, K. 1991, A\&A, 244, 437

Werner, K., Dreizler, S., Heber, U., et al. 1996, A\&A, 307, 860

Werner, K., Deetjen, J. L., Dreizler, S., et al. 2003, in Stellar Atmosphere Modeling, eds. I. Hubeny, D. Mihalas, \& K. Werner, ASP Conf. Ser., 288, 31

Werner, K., Rauch, T., Reiff, E., Kruk, J. W., \& Napiwotzki, R. 2004, A\&A, 427, 685

Werner, K., Rauch, T., \& Kruk, J. W. 2005, A\&A, 433, 641

Werner, K., Rauch, T., \& Kruk, J. W. 2007a, A\&A, 466, 317

Werner, K., Rauch, T., \& Kruk, J. W. 2007b, A\&A, 474, 591

Werner, K., Rauch, T., \& Kruk, J. W. 2010, ApJ, 719, L32

Werner, K., Rauch, T., Kruk, J. W., \& Kurucz, R. L. 2011, A\&A, 531, A146

Werner, K., Rauch, T., \& Kepler, S. O. 2014, A\&A, 564, A53

Werner, K., Rauch, T., \& Kruk, J. W. 2015, A\&A, 582, A94

Wesemael, F., Green, R. F., \& Liebert, J. 1985, ApJS, 58, 379 